\title{
Instantaneous charge separation in non- fullerene acceptor bulk-heterojunction of highly efficient solar cells
}

\author{
Franco V. A. Camargo ${ }^{1, *}$, Nicola Gasparini ${ }^{2}$, Tetsuhiko Nagahara $^{1,3}$, Larry Lüer $^{4}$, Giulio \\ Cerullo $^{1}$, and Christoph Brabec $^{2,5}$ \\ ${ }^{1}$ IFN-CNR, Dipartimento di Fisica, Piazza Leonardo da Vinci 32, 20133 Milano, Italy \\ ${ }^{2}$ Institute of Materials for Electronics and Energy Technology (I-MEET), Friedrich Alexander- \\ University Erlangen-Nuremberg, Martensstraße 7, 91058 Erlangen, Germany \\ ${ }^{3}$ Kyoto Institute of Technology, Department of Chemistry and Materials Technology, Kyoto, Japan \\ ${ }^{4}$ Madrid Institute for Advanced Studies, IMDEA Nanoscience, Calle Faraday 9 , \\ Campus Cantoblanco, 28049 Madrid, Spain \\ ${ }^{5}$ Bavarian Center for Applied Energy Research (ZAE Bayern), Haberstrasse 2a, 91058 Erlangen, \\ Germany
}

\begin{abstract}
Using broadband transient absorption in a high efficiency $(>11 \%)$ photovoltaic blend with a non-fullerene acceptor, we observe instantaneous (sub-30 fs) charge separation, demonstrating close to ideal donor-acceptor level matching and nanomorphology in this blend.
\end{abstract}

The field of organic photovoltaics is experiencing a surge in efficiency for devices based on bulk-heterojunctions that include non-fullerene acceptors [1]. These can be specially designed to provide both complementary light absorption and optimal driving force for charge separation, thus at the same time maximizing open circuit voltage (by avoiding losses due to excess driving force) and short circuit current (maximising light absorption and charge separation yields). Here, we investigate the ultrafast charge separation dynamics of one such high performance photovoltaic blend.

The $o$-IDTBR (Fig. 1a) is a novel electron acceptor with scalable synthesis, tailor made to provide good morphology, charge transport properties and increased absorption in the red and near infrared (NIR) spectral regions. Devices with $o$-IDTBR and the most readily available polymer $\mathrm{P} 3 \mathrm{HT}$ have shown record $6.4 \%$ power conversion efficiency (PCE) among P3HT devices with non-fullerene acceptors [2], going up to $11 \%$ when a mix of $o$ IDTBR and its variant EH-IDTBR were combined with the polymer PCE10 [3]. In both cases improved stability compared to fullerene-based devices was also observed. Here we study the charge separation process of $o$-IDTBR blended with another polymer, WF3 (Fig. 1b), for which devices with PCE higher than $11 \%$ were obtained.

\footnotetext{
* Corresponding author: francovalduga@gmail.com
} 


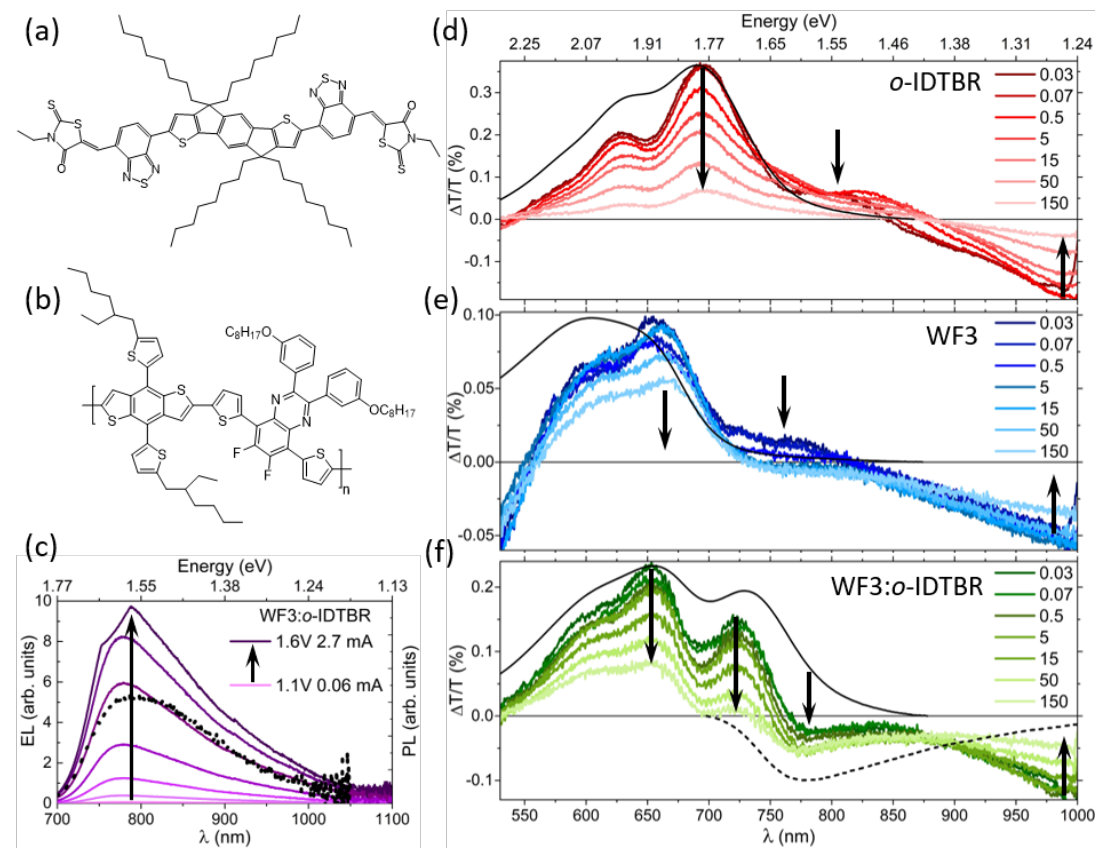

Fig. 1. Molecular structure of (a) o-IDTBR and (b) WF3. (c) EL of WF3:o-IDTBR bulkheterojunction for different injected currents (solid lines), and PL of the bulk-heterojunction (black dots). (d) Sequence of chirp-corrected TA spectra of pristine $o$-IDTBR from 0.03 to $150 \mathrm{ps}$ and linear absorption spectrum (black). (e) Same as (d) for WF3. (f) Same as (d) for the WF3:oIDTBR blend. The dashed line in (f) is the electroluminescence multiplied by a negative scaling factor for comparison with the PIA band.

The linear absorption spectra of $o$-IDTBR, WF3 and the blend are shown in solid black lines in Figures 1d, 1e and 1f respectively. In the blends the $o$-IDTBR peak at $690 \mathrm{~nm}$ is redshifted to $729 \mathrm{~nm}(775 \mathrm{~cm}-1$ or $96 \mathrm{meV}$ shift), suggesting that a different crystalline domain of the $o$-IDTBR is formed near the interface with the WF3, potentially involving wavefunction delocalization between them. One notable feature of the WF3:o-IDTBR devices is that they show electroluminescence (EL) quantum yields nearly two orders of magnitude higher than the typical values for polymers blended with fullerene acceptors (including WF3). In Figure 1c the EL spectrum of WF3:o-IDTBR for different injected currents is shown in shades of purple, and it matches the photoluminescence (PL) spectrum of the WF3:o-IDTBR (black dots in Fig. 1c). Thus, injecting either electrons or photons in the WF3:o-IDTBR bulk-heterojunction both trigger emission from the same NIR band, supporting the hypothesis that this band corresponds to an emissive charged state. Further, the PL of WF3:o-IDTBR is quenched compared to that of the pristine materials, suggesting that this charged state is localized at the interface, with the PL being quenched due to diffusion of most of the separated carriers into the donor and acceptor domains.

Broadband transient absorption (TA) spectroscopy was performed using $\approx 10 \mathrm{fs}$ excitation pulses with spectrum spanning from 525 to $730 \mathrm{~nm}$ generated with a noncollinear OPA, keeping the samples under nitrogen and pump intensities between 1 and $4 \mu \mathrm{J} / \mathrm{cm}^{2}$ to avoid bimolecular recombination kinetics [4]. In order to probe the spectral region of main interest (between 730 and $850 \mathrm{~nm}$ ) continuously, we built a narrowband OPA at $1225 \mathrm{~nm}$ 
pumped with an $800 \mathrm{~nm} \mathrm{1-kHz} \mathrm{Ti:Sapphire} \mathrm{laser} \mathrm{system,} \mathrm{yielding} \mathrm{a} \mathrm{white} \mathrm{light} \mathrm{continuum}$ from 540 to $1000 \mathrm{~nm}$ with RMS shot-to-shot fluctuations below $0.3 \%$ throughout the spectrum.

In Figures 1d, 1e and 1f we plot the chirp-corrected TA spectra between 0.03 and $150 \mathrm{ps}$ of $o$-IDTBR, WF3 and the bulk-heterojunction respectively. For $o$-IDTBR (Fig. 1d), the TA spectra are dominated by the singlet exciton, shown by nearly equal strength of the $(0,1)$ and $(1,0)$ vibronic transitions of photobleach (PB) and stimulated emission (SE), respectively, at $t>1$ ps when spectral relaxation is complete. For pure WF3 (fig. 1e), we observe PB vibronic features in agreement with the respective ground state absorption bands and a very small sub-bandgap signal around $770 \mathrm{~nm}$ with only a few hundred fs lifetime, consistent with internal conversion of the initial, optically allowed singlet state to a dark one.

In contrast, the TA spectra of the blend (Fig. 1f) shows a PIA band peaking at 780 $\mathrm{nm}$ since the earliest times, which is not present in the pristine materials. This band has the same spectral characteristics as the EL band measured in the 11\% PCE devices, which is plotted in a dashed line multiplied by a negative factor in Figure $1 \mathrm{f}$ to ease comparison. The appearance of this band from time zero (within the $\approx 30$ fs instrumental response function) in the broadband TA spectra confirms the hypothesis that an interfacial charged state is present in this system. Furthermore, even in timescales of tens of picoseconds this PIA band continues to rise as the PIA from the pristine materials at $1000 \mathrm{~nm}$ decays, consistent with exciton diffusion in such systems [5,6]. This suggests that absorption near the bulk-heterojunction interface yields instantaneous charge separation, but electron and hole transfer processes still occur on longer timescales when the photoexcitation happens deep within the donor or the acceptor domains. Finally, we employ TA experiments with narrowband excitation at $730 \mathrm{~nm}$ (selective to the $o$-IDTBR) at different fluences to completely characterize the hole transfer dynamics in this system.

\section{References}

1. J. Hou, O. Inganäs, R. H. Friend, F. Gao, Nat. Mater. 17, 119-128 (2018).

2. S. Holliday, R. S. Ashraf, et al., Nat. Commun. 7, 11585 (2016).

3. D. Baran, R. S. Ashraf, et al., Nat. Mater. 16, 363-369 (2017).

4. J. Piris, T. E. Dykstra, et al., J. Phys. Chem. C 113, 14500-14506 (2009).

5. H. Bin, L. Gao, et al., Nat. Commun. 7, 13651 (2016).

6. J. Shi, A. Isakova, et al., Energy Environ. Sci. 11, 211 (2018). 